\title{
Robust method for distinguishing heterozygous from homozygous transgenic alleles by multiplex ligation-dependent probe assay
}

\author{
Piotr Kozlowski, Mei Lin, Lynsey Meikle, and David J. Kwiatkowski \\ Harvard Medical School, Boston, MA, USA
}

BioTechniques 42:584-588 (May 2007)

doi $10.2144 / 000112473$

Transgenic mouse alleles continue to be used heavily in biomedical research (1). Transgenes insert into the genome at random sites, typically in a tandem array of 1 to 20 copies. Both Southern blot analysis and real-time PCR can be used for determining the zygosity of transgenes, but each have practical and technical limitations (2-4). Here we describe a robust, easily implemented method for determination of zygosity of transgene alleles, which gives a clear, reproducible distinction between 1 versus 2 alleles. The method uses the multiplex ligation-dependent probe assay (MLPA) with a competitor oligonucleotide. MLPA is a widely used method for assessing the relative copy number of multiple genomic sequences in a DNA sample (5). During MLPA, an oligonucleotide ligation reaction is performed, followed by PCR using

Figure 1. Design of transgene-specific probe sets for multiplex ligation-dependent probe assay (MLPA), competitor oligonucleotides, and MLPA electropherograms and graphs. (A) Diagram of 5' and $3^{\prime}$ half-probes hybridizing to their target sequence (left) and competed from binding by competitor oligonucleotide without primer specific and stuffer sequences (right). (B) Electropherograms of heterozygous (+/-, left panels) and homozygous (+/+, right panels) SynICre DNA samples with varying ratios of $5^{\prime}$ half-probe to competitor (from top to bottom: 1:0, 1:1, 1:2). Note the high ratio between the cre 1 peak and the control peaks in the absence of competitor, and that the peak for each of the Cre probe sets (cre1, cre2) becomes smaller as the proportion of competitor is increased. (C) Representation of peak height ratios. Using the same data as in panel B, the $\mathrm{x}$-axis is percentage competitor relative to transgene-specific $5^{\prime}$ probe (100\% corresponds to $1: 1$ in panel $B$ ). The $y$-axis values are the peak height for the transgene probe divided by the average peak height for the control probes. Signals for homozygote and heterozygote are shown in blue and red color, respectively. a fluorescein-conjugated primer, such that the amount of PCR product generated for each genomic sequence is directly proportional to the number of input copies (Figure 1A, left).

For this MLPA assay, genomic DNA samples were isolated from mouse tail or toe snips using the Puregene ${ }^{\circledR}$ Tail DNA method (Gentra, Minneapolis,
MN, USA) and adjusted to approximately $50 \mathrm{ng} / \mu \mathrm{L}$. Mice bearing four different Cre transgene alleles were studied: SynICre (6), TetOpCre (7), Nestin-Cre, and Wnt-Cre, as well as two other transgene alleles: the Sleeping Beauty transposon T2/Onc (8) and enhanced green fluorescent protein (EGFP) (7).

For each of the three transgenes analyzed, two MLPA probe sets were designed (see Supplementary Table S1 available online at www.BioTechniques. com) to have amplification products of size approximately $120 \mathrm{bp}$ and approximately $170 \mathrm{bp}$. Three control probes elsewhere in the mouse genome were used, with amplification products ranging in size from 108 to $136 \mathrm{bp}$. Each probe set was composed of a $5^{\prime}$ and a $3^{\prime}$ half-probe, each containing unique target specific sequence, stuffer sequence, and universal primer
A
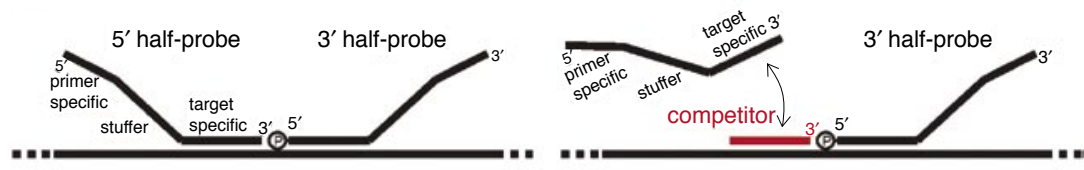

B
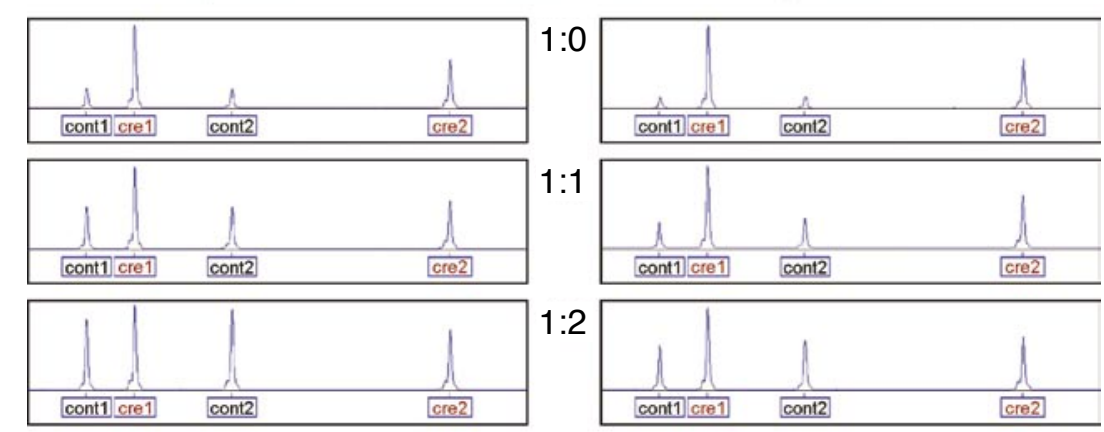

\section{C}

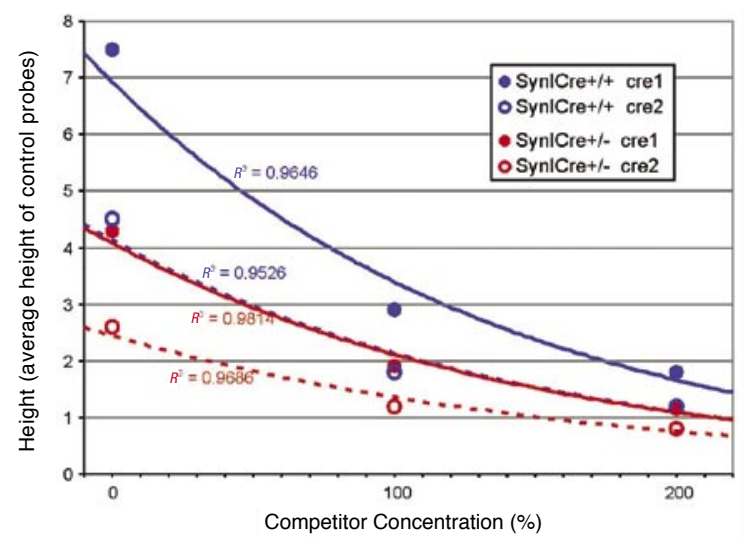




\section{Benchmarks}

A

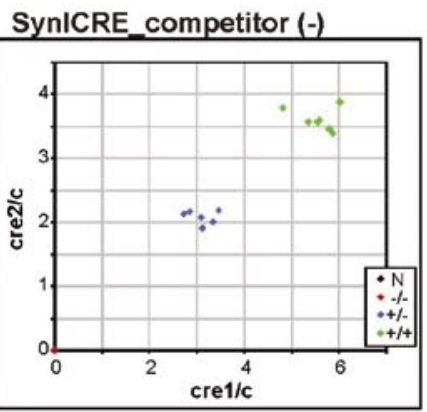

TetOpCRE_competitor (-)

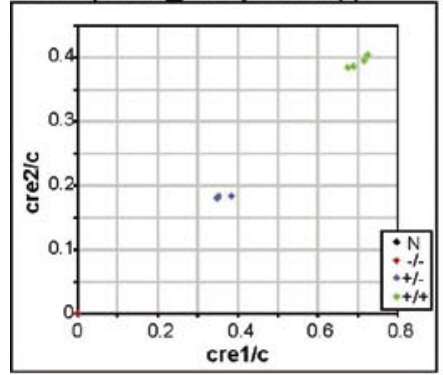

Wnt-CRE_competitor (+)

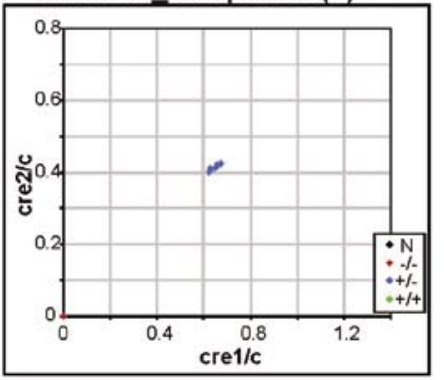

Nestin-CRE_competitor (+)

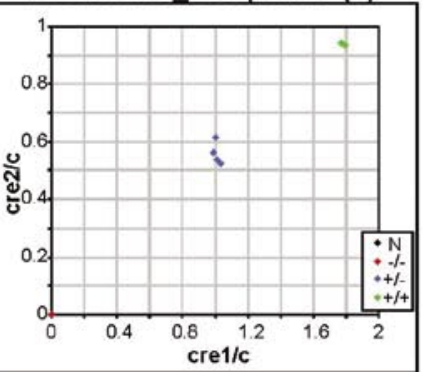

B

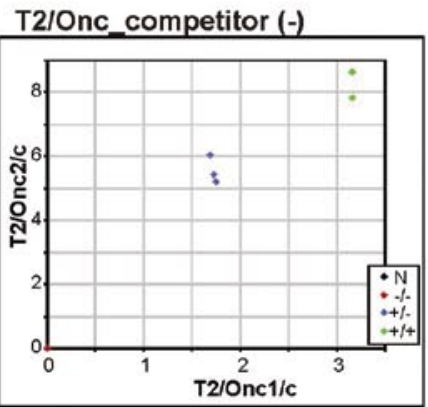

EGFP_competitor (-)

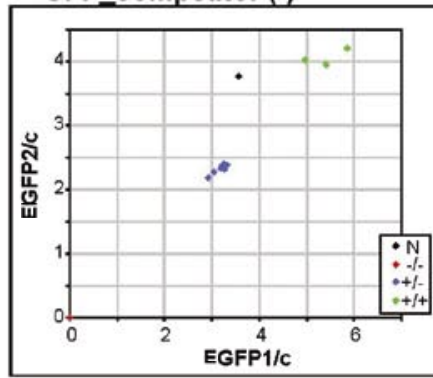

EGFP_competitor (+)

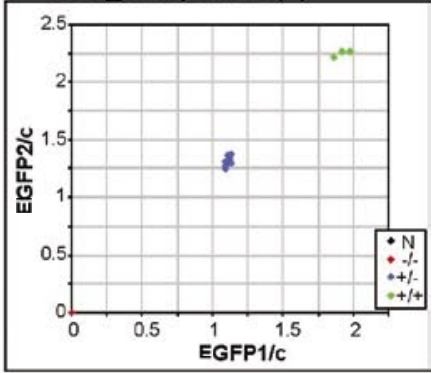

Figure 2. Scatter plot analysis of multiplex ligation-dependent probe assay (MLPA) results for genotyping transgene alleles. (A) Cre transgenes. (B) T2/Onc transgene. (C) Enhanced green fluorescent protein (EGFP) transgene. $x$ - and $y$-axis values are relative signals for each of two transgene-specific probe sets. Presence or absence of competitor is indicated. Panel C provides a direct comparison of the results obtained without and with competitor and shows that the scatter plot in which competitor was added was more tightly clustered. Note that there are no homozygous $(+/+)$ samples for the Wnt-Cre allele, and that multiple homozygous (-/-) samples were assessed for each transgene, but all had uniform values of $(0,0)$ on these graphs, so that a single red dot is seen at $(0$, $0)$ in each scatter plot.

sequences on their $5^{\prime}$ and $3^{\prime}$ ends, respectively $(5,9)$ (Supplementary Table S1 and Figure 1A). All probes were synthesized at $100 \mathrm{nM}$ scale and purified by polyacrylamide gel electrophoresis (PAGE; Integrated DNA Technologies, Skokie, IL, USA). 3' half-probes were synthesized with a $5^{\prime}$ phosphate to facilitate ligation.

MLPA was performed as described previously $(5,9)$. Briefly, $250 \mathrm{ng}$ DNA in $5 \mu \mathrm{L}$ were incubated at $98^{\circ} \mathrm{C}$ for 5 min; after cooling to room temperature, it was mixed with $1.5 \mu \mathrm{L}$ transgenespecific probes mixture (containing $1.5 \mathrm{fmol}$ each probe and $0-3 \mathrm{fmol}$ each competitor-probe) and $1.5 \mu \mathrm{L}$ SALSA hybridization buffer, then denatured at $95^{\circ} \mathrm{C}$ for $2 \mathrm{~min}$ and hybridized at $60^{\circ} \mathrm{C}$ for $16 \mathrm{~h}$. Hybridized probes were then ligated at $54^{\circ} \mathrm{C}$ for $15 \mathrm{~min}$ by addition of $32 \mu \mathrm{L}$ ligation mixture. Following heat inactivation, $10 \mu \mathrm{L}$ ligation reaction was mixed with $30 \mu \mathrm{L}$ PCR buffer, heated to $60^{\circ} \mathrm{C}$, mixed with 10 $\mu \mathrm{L}$ PCR mixture (SALSA polymerase, dNTPs, and universal primers, one of which was labeled with fluorescein), and subjected to PCR amplification for 30 cycles. All reagents except probe mixes were from MRC-Holland (Amsterdam, The Netherlands).

Amplification products were diluted in water and then 1:9 in Hi$\mathrm{Di}^{\mathrm{TM}}$ formamide (Applied Biosystems, Foster City, CA, USA) containing 1/36 volume of $500 \mathrm{ROX}^{\mathrm{TM}}$ size standard (Applied Biosystems), to a final dilution of 20- to 200-fold, and then were separated by size on an ABI PRISM ${ }^{\circledR}$ 3100 Genetic Analyzer (Applied Biosystems). Electropherograms were analyzed by GeneMapper ${ }^{\circledR}$ v3.5 (Applied Biosystems), and peak height data were exported to an Excel $^{\circledR}$ table.

MLPA analysis for three different transgene sequences demonstrated that the signal intensity (peak height) from the transgenic allele was typically much higher than that seen with single-copy sequences (Figure 1B, top $1: 0)$. For example, the ratio of the peak heights for two different homozygous Cre recombinase transgenes studied were 6.5 and 5.4 for one Cre probe set (cre1) and 3.5 and 3.3 for a second, longer Cre probe set (cre2), respectively. This ratio reflects the copy number of Cre sequences in each of these transgenes.

Normalization of peak height data was done by dividing each transgene peak height by the average signal from three control probes, followed by division by a similar value calculated from a set of reference samples known to be heterozygotes for the transgene allele. This calculation demonstrated that all three categories of transgene genotype, homozygous absent, heterozygous, and homozygous present, were usually easily distinguished in a blinded analysis of multiple samples for the six different transgenes of three different types (Supplementary Figure S1). Results were confirmed for one allele (SynICre) in a blinded manner by comparison with genotype status as determined by breeding, with perfect concordance.

However, the high ratio of the transgene to control peak heights for most transgenes (five of six studied here) led to difficulty in two ways. First, repeated capillary runs at different dilutions were often required to achieve signal intensity within the dynamic 


\section{Benchmarks}

range of quantitative detection for the peaks being analyzed. Second, highly unequal probe intensities appeared to lead to higher variability in peak ratio determination.

Therefore, we explored the utility of competitor probes to selectively reduce signal from the transgene allele probe sets. Competitor oligonucleotides were identical to the transgene allele $5^{\prime}$ half-probes, but lacked the stuffer and primer specific sequences, so that they would not be amplified in the PCR step of the MLPA procedure (Figure 1 A right and Supplementary Table S1). Inclusion of competitor oligonucleotide at a $1: 1$ or $2: 1$ ratio to the transgenespecific MLPA oligonucleotide probe led to a proportional decrease in the peak heights from the transgene alleles relative to control probe sets, as expected (Figure 1, B and C).

Competitor oligonucleotides were then used for the analysis of five transgene alleles that had a high copy number. In each case, the competitor used at a ratio of $2: 1$ brought the transgene allele peak height down to values similar to those of control alleles. To further simplify interpretation of allele signals and distinguish heterozygotes from homozygotes, we created a scatter plot for all samples analyzed for a single transgene (Figure 2). The $\mathrm{x}$ axis value on the scatter plot came from one transgene-specific probe set, and the $y$-axis value came from the other transgene-specific probe set. Individual DNA samples were observed to form three tight clusters when plotted in this way, which corresponded to the three possible genotypes. Notably, these clusters were more compact when competitor oligonucleotides were used, making peak heights more similar and enabling higher confidence in genotype assignment. To formally test this impression, a comparison was made between MLPA without competitor and with a 2:1 competitor ratio for analysis of the EGFP transgene. The standard deviations for normalized signals from heterozygotes and homozygotes were 0.120 and 0.088 without competitor, and 0.021 and 0.038 with competitor (Figure 2), indicating the value of use of the competitor.

In summary, we have developed a robust MLPA method for genotyping of transgene alleles, for six different transgenes of three different types. The method is inexpensive and fast and can be implemented in any molecular DNA laboratory with relative ease, particularly those with MLPA experience. It should prove useful in genotyping the vast number of transgene alleles in common use and is a desirable alternative to other methods of determining zygosity.

\section{ACKNOWLEDGMENTS}

This work was supported by National Institutes of Health (NIH) grant no. NS31535. The authors thank Dawn Ciulla for technical assistance.

\section{COMPETING INTERESTS STATEMENT}

The authors declare no competing interests.

\section{REFERENCES}

1.Schneider, M.R. and E. Wolf. 2005. Genotyping of transgenic mice: old principles and recent developments. Anal. Biochem. 344:1-7.

2. Ballester, M., A. Castello, E. Ibanez, A. Sanchez, and J.M. Folch. 2004. Real-time quantitative PCR-based system for determining transgene copy number in transgenic animals. BioTechniques 37:610-613.

3.Shitara, H., A. Sato, J. Hayashi, N. Mizushima, H. Yonekawa, and C. Taya. 2004. Simple method of zygosity identification in transgenic mice by real-time quantitative PCR. Transgenic Res. 13:191-194.

4. Tesson, L., J.M. Heslan, S. Menoret, and I. Anegon. 2002. Rapid and accurate determination of zygosity in transgenic animals by real-time quantitative PCR. Transgenic Res. 11:43-48.

5. Schouten, J.P., C.J. McElgunn, R. Waaijer, D. Zwijnenburg, F. Diepvens, and G. Pals. 2002. Relative quantification of 40 nucleic acid sequences by multiplex ligation-dependent probe amplification. Nucleic Acids Res. 30:e57.

6.Zhu, Y., M.I. Romero, P. Ghosh, Z. Ye, P. Charnay, E.J. Rushing, J.D. Marth, and L.F. Parada. 2001. Ablation of NF1 function in neurons induces abnormal development of cerebral cortex and reactive gliosis in the brain. Genes Dev. 15:859-876.

7. Yu, T.S., M. Dandekar, L.M. Monteggia, L.F. Parada, and S.G. Kernie. 2005. Temporally regulated expression of Cre re- combinase in neural stem cells. Genesis 41:147-153.

8. Collier, L.S., C.M. Carlson, S. Ravimohan, A.J. Dupuy, and D.A. Largaespada. 2005 Cancer gene discovery in solid tumours using transposon-based somatic mutagenesis in the mouse. Nature 436:272-276.

9. Kozlowski, P., P. Roberts, S. Dabora, D. Franz, J. Bissler, H. Northrup, K.S. Au, R. Lazarus, et al. 2007. Identification of 54 large deletions/duplications in TSC1 and TSC2 using MLPA, and genotype-phenotype correlations. Hum. Genet. Feb 8; [Epub ahead of print].

Received 23 February 2007; accepted 21 March 2007.

Address correspondence to David J. Kwiatkowski, Brigham and Women's Hospital, One Blackfan Circle, Rm 6-216, Boston, MA 02115, USA e-mail:dk@rics.bwh.harvard.edu

To purchase reprints of this article, contact: Reprints@BioTechniques.com 